\title{
Commentary Left ventricle restoration. Keep the door open.
}

\author{
Marco Moscarelli ${ }^{1}$ and Carlo Olevano ${ }^{2}$ \\ ${ }^{1}$ GVM Care and Research \\ ${ }^{2}$ Southampton General Hospital
}

September 25, 2021

\begin{abstract}
Surgical left ventricle restoration (SVR) was firstly by Cooley in 1958 with the "linear suture technique", and three decades later, Dor used a circular patch to reconstruct the left ventricle excluding the scarred parts of the septum and ventricular wall. It gained popularity and eventually almost abandoned after the contrasting literature evidences. Hassanabad et al. presented a comprehensive review of current literature on surgical ventricle restoration (SVR) techniques and clinical outcomes, trying to understand if SVR has still a substantial role in the modern medicine.
\end{abstract}

\section{Commentary}

\section{Left ventricle restoration. Keep the door open.}

Carlo Olevano ${ }^{1}$, Marco Moscarelli ${ }^{2}$

1 Moscati Hospital, Dept. of Cardiovascular surgery, Avellino, Italy

2 Anthea Hospital, GVM Care \& Research, Dept. of Cardiovascular surgery Bari, Italy

Word count (all): 1614

Disclosure: none.

\section{Abstract}

Surgical left ventricle restoration (SVR) was firstly by Cooley in 1958 with the "linear suture technique", and three decades later, Dor used a circular patch to reconstruct the left ventricle excluding the scarred parts of the septum and ventricular wall. It gained popularity and eventually almost abandoned after the contrasting literature evidences. Hassanabad et al. presented a comprehensive review of current literature on surgical ventricle restoration (SVR) techniques and clinical outcomes, trying to understand if SVR has still a substantial role in the modern medicine.

The worldwide human and economic burden of congestive heart failure (HF) has rapidly grown in the last two decades with an estimated overall cost of $\$ 108$ billons per annum with $\$ 65$ billons attributed to direct expense accounted for by hospitalization and $\$ 48$ billons to indirect costs; US is responsible for $28.4 \%$ of total spend while Europe account for $6.8 \%$ (1).

Heart transplantation remains the treatment of choice for patients with medically refractory end-stage HF, nevertheless the need for immunosuppression and the limited donor supply have restricted the selection criteria, leaving physicians seeking for alternative therapies $(2,3)$.

Hassanabad et al. presented a comprehensive review of current literature on surgical ventricle restoration (SVR) techniques and clinical outcomes, trying to understand if SVR has still a substantial role in the modern medicine. They also critically analysed data on left ventricle morphology and size, mitral valve disfunction 
and arrhythmias (4). Cardiac remodelling is generally accepted as a determinant of the clinical course of HF and comprises changes in left ventricle cavity diameters, mass, geometry and function. As early as few hours after heart injury, the initial remodelling mechanism leads to reparation of necrotic area through cellular rearrangement of the ventricular wall in order to preserve cardiac output. As the heart remodels, the left ventricle increase in diameter and become less elliptical and more spherical (5). The rationale behind surgical ventricular restoration, as we know, is to reverse left ventricle remodelling, restoring a more physiological heart geometry and and improving mitral valve functioning reducing left ventricle diameter and papillary muscle distances.

This "correction" was firstly described by Cooley in 1958 with the "linear suture technique", while three decades later, Dor used a circular patch to reconstruct the left ventricle excluding the scarred parts of the septum and ventricular wall. These techniques were adopted and modified by many surgeons in the modern era $(6,7)$.

Data on the performance of SVR are not univocal. The surgical Treatment for Ischemic Heart Failure (STICH) Trial found that the addition of SVR to coronary artery bypass grafting (CABG) reduce the endsystolic volume index significantly compared with CABG alone; however this anatomical conversion was not associated with a more favourable death-rate or hospitalization for cardiac causes. Nonetheless, SVR may remain a valuable strategy combined with CABG in selected HF patients with a scar in the left anterior descending artery territory, especially if a post-operative left ventricle end systolic volume (LVESV) index $<70 \mathrm{~mL} / 2$ can be achieved, as recently reported by European Society of Cardiology (ESC) and European Association for Cardio- Thoracic Surgery (EACTS) (Class of Recommendation IIb; level of evidence B) $(8,9)$.

Ischemic mitral regurgitation (IMR) is a frequent complication of chronic ischemic heart disease. In the failing heart, IMR occurs duo to annular dilatation secondarily to left ventricle and papillary muscles morphology and functional changes (10). The effects of mitral valve surgery in patients with significant IMR in the setting of $\mathrm{HF}$ is still controversial. H Wu et al. didn't find any improvement in long-term survival among more than one hundred patients with severe left ventricle dysfunction that underwent mitral valve annuloplasty (11).

More recently the Randomized Ischemic Mitral Evolution (RIME) trial and POINT trial demonstrated the efficacy of adding valve repair to CABG on ventricular remodelling, ejection fraction, symptoms and degree of mitral regurgitation but not with regard to mortality $(12,13,14)$.

The prognostic stratification of patients with ischemic cardiomyopathy undergoing SVR is a potential area of future study and improvement for surgical intervention. In this direction, Toso et al. demonstrated that patients who underwent SVR with an elevated level of N-terminal pro-B-type natriuretic peptide combined with presence of restrictive filling pattern had the worst outcome in terms of mortality, hospitalization for $\mathrm{HF}$ and major adverse cardiac events (MACE) at 36 months (15).

Magnetic resonance imaging (MRI) has been gaining popularity in the context of HF, given the potential to identify the ideal candidates for SVR procedure. Yamazaki et al. confirmed the importance of preoperative LVESV index as a predictor of MACE following SVR. Patient with preoperative LVESV index of $>130 \mathrm{ml}^{2}$ had worst outcome compared to intermediate (LVESV index 100 to $130 \mathrm{ml}^{2}$ ) and lower (LVESV index $<100$ $\mathrm{ml}^{2}$ ) group (16).

Right ventricle volume along function MRI assessment may also have predictive value in the risk stratification of SVR. (17). As highlighted by Hassanabad et al., the study of left ventricle myofiber orientation and stress is now possible thanks the advance on MRI technology and mathematical modelling. After successful SVR and aneurysm plication, left ventricle myofibers orientation is significantly rearranged resulting in a more uniform stress distribution and subsequently reduced oxygen demand. Notably, given the most recent developments, MRI technology is definitely a promising field of research in SVR prognostic stratification.

Surgical restoration remains a complex procedure that and needs multi-disciplinary approach. As such should be performed in high-volume and specialized centres. However, there are evidences to suggest that it might be a viable option for eligible / selected cardiac transplant patients. 


\section{Glossary}

$\mathbf{C A B G}=$ Coronary artery bypass grafting

$\mathbf{H F}=$ Heart failure

$\mathbf{I M R}=$ Ischemic mitral regurgitation

LVESV = Left ventricle end systolic volume

MACE $=$ Major adverse cardiac events

MRI= Magnetic resonance imaging

STICH $=$ The surgical Treatment for Ischemic Heart Failure

$\mathbf{S V R}=$ Surgical ventricle restoration

\section{References}

1. Cook C, Cole G, Asaria P, Jabbour R, Francis DP. The annual global economic burden of heart failure. Int J Cardiol. 2014 Feb 15;171(3):368-76. doi: 10.1016/j.ijcard.2013.12.028. Epub 2013 Dec 22. PMID: 24398230 .

2. Copeland JG, McCarthy M. University of arizona, cardiac transplantation: changing patterns in selection and outcomes. Clin Transpl 2001: 203-7.

3. Deuse T, Haddad F, Pham M, et al . Twenty-year survivors of heart transplantation at stanford university. Am J Transplant 2008; 8: 1769-74

4. Clinical and Hemodynamic Outcomes of the Dor Procedure in Adults with Ischemic Cardiomyopathy. Ali Fatehi Hassanabad, Kiana Wiebe, Imtiaz Ali 2021. JOCS. In press.

5. Cohn JN, Ferrari R, Sharpe N. Cardiac remodeling-concepts and clinical implications: a consensus paper from an international forum on cardiac remodeling. Behalf of an International Forum on Cardiac Remodeling. J Am Coll Cardiol. 2000 Mar 1;35(3):569-82. doi: 10.1016/s0735-1097(99)00630-0. PMID: 10716457 .

6. Cooley DA, Collins HA, Morris GC Jr, Chapman DW. Ventricular aneurysm after myocardial infarction. Surgical excision with the use of temporary cardiopulmonary bypass. JAMA 1958; 167: 557- 60.

7. Dor V, Saab M, Coste P, Kornaszewska M, Montiglio F. Left ventricular aneurysm: a new surgical approach. J Thorac Cardiovasc Surg 1989; 37: 11-9.

8. JonesRH, VelazquezEJ, MichlerRE, SopkoG, OhJK, O'ConnorCM, etal.Coronary bypass surgery with or without surgical ventricular reconstruction. N Engl J Med. 2009;360:1705-17.

9. Neumann FJ, Sousa-Uva M, Ahlsson A, Alfonso F, Banning AP, Benedetto U, et al. [2018 ESC/EACTS guidelines on myocardial revascularization. The task force on myocardial revascularization of the European Society of Cardiology (ESC) and European Association for Cardio- Thoracic Surgery (EACTS)]. G Ital Cardiol (Rome). 2019;20(Suppl 1): 1S-61S (in Italian).

10. Levine RA. Dynamic mitral regurgitation: more than meets the eye. N Engl J Med 2004; 351: 1681-4.

11. Wu AH, Aaronson KD, Bolling SF, Pagani FD, Welch K, Koelling TM. Impact of mitral valve annuloplasty on mortality risk in patients with mitral regurgitation and left ventricular systolic dysfunction. J Am Coll Cardiol. 2005 Feb 1;45(3):381-7. doi: 10.1016/j.jacc.2004.09.073. PMID: 15680716.

12. Deja MA, Grayburn PA, Sun B, Rao V, She L, Krejca M et al. Influence of mitral regurgitation repair on survival in the surgical treatment for is- chemic heart failure trial. Circulation. 2012;125:2639- 2648. 
13. Chan KM, Punjabi PP, Flather M, Wage R, Symmonds K, Roussin I, Rahman-Haley S, Pennell DJ, Kilner PJ, Dreyfus GD, Pepper JR; RIME Investigators. Coronary artery bypass surgery with or without mitral valve annuloplasty in moderate functional ischemic mitral regurgitation: final results of the Randomized Ischemic Mitral Evaluation (RIME) trial. Circulation. 2012 Nov 20;126(21):2502-10. doi: 10.1161/CIRCULATIONAHA.112.143818. Epub 2012 Nov 7. PMID: 23136163.

14. Fattouch K, Guccione F, Sampognaro R, Panzarella G, Corrado E, Navarra E, Calvaruso D, Ruvolo G. POINT: Efficacy of adding mitral valve restrictive annuloplasty to coronary artery bypass grafting in patients with moderate ischemic mitral valve regurgitation: a randomized trial. J Thorac Cardiovasc Surg. 2009 Aug;138(2):278-85. doi: 10.1016/j.jtcvs.2008.11.010. Epub 2009 May 31. PMID: 19619766.

15. Toso A, Castelvecchio S, Menicanti L, Volpe M, Fantini F. Prognostic value of natriuretic peptides and restrictive filling pattern before surgical ventricular restoration. J Thorac Cardiovasc Surg. 2020 Oct 15:S0022-5223(20)32827-0. doi: 10.1016/j.jtcvs.2020.09.132. Epub ahead of print. PMID: 33168168.

16. Yamazaki S, Doi K, Numata S, Itatani K, Kawajiri H, Morimoto K, Manabe K, Ikemoto K, Yaku $\mathrm{H}$. Ventricular volume and myocardial viability, evaluated using cardiac magnetic resonance imaging, affect long-term results after surgical ventricular reconstruction. Eur J Cardiothorac Surg. 2016 Oct;50(4):704-712. doi: 10.1093/ejcts/ezw213. Epub 2016 Jun 28. PMID: 27354255.

17. Yamazaki S, Numata S, Inoue T, Itatani K, Morimoto K, Ohira S, Manabe K, Yokota I, Yaku H. Impact of right ventricular volume and function evaluated using cardiovascular magnetic resonance imaging on outcomes after surgical ventricular reconstruction. Eur J Cardiothorac Surg. 2018 Nov 1;54(5):867-874. doi: 10.1093/ejcts/ezy189. PMID: 29788059. 